\title{
O uso do GeoGebra no ensino de trigonometria: uma experiência com alunos do ensino médio
}

\author{
The use of GeoGebra in teaching trigonometry: an experience with school students
}

\author{
Aleksandre Saraiva Dantas \\ Instituto Federal de Educação, Ciência e Tecnologia do Rio Grande do Norte, RN, Brasil
}

\begin{abstract}
Resumo
Essa pesquisa busca analisar se o trabalho com o GeoGebra facilita a aprendizagem de conceitos da trigonometria e conhecer as percepções dos alunos acerca do uso do GeoGebra no ensino de trigonometria. Para isso, faz uso da aplicação de avaliações e entrevistas com os alunos do IFRN. A análise dos dados mostra que, com o uso do GeoGebra, os alunos apresentaram uma aprendizagem mais significativa de diversos aspectos do comportamento das funções seno e cosseno, quando comparado com o ensino através de aulas expositivas com o uso de recursos didáticos mais comuns, como o livoro e o quadro branco. Os alunos reconheceram que o uso desse software ajuda a compreender melhor os aspectos inerentes ao comportamento dessas funções, ressaltando a facilidade na utilização do GeoGebra e a importância da sua utilização no ensino de Matemática. Para que os professores se sintam capazes de trabalhar com o GeoGebra ou com qualquer outro software, é fundamental que os cursos de formação de professores desenvolvam estratégias que garantam, efetivamente, a capacidade de utilização dessas ferramentas, de forma crítica e criativa, e não apenas como mais um modismo que em nada irá ajudar a melhorar o trabalho do professor e a aprendizagem dos alunos.
\end{abstract}

Palavras-chave: GeoGebra. Ensino. Aprendizagem. Alunos. Trigonometria

\begin{abstract}
This research analyzes if the work with GeoGebra facilitates learning concepts of trigonometry and knowing the perceptions of students about the use of GeoGebra in teaching trigonometry. For this, it makes use of application of assessments and interviews with the students of courses of IFRN. Data analysis shows that, with the use of GeoGebra, students showed more significant learning of several aspects of the behavior of the sine and cosine functions when compared with teaching through lectures with the use of teaching resources more common as book and whiteboard. The students themselves recognized that the use of this software is able to help you better understand the aspects of the behavior of these functions, highlighting the ease of use of GeoGebra and the importance of its use in teaching mathematics. However, for the current and future teachers feel able to work with GeoGebra or any other software, it is essential that the vocational training courses for teachers to develop strategies that ensure effectively the ability to use these tools, the critically and creatively way, and not just another fad that will in no way help improve the teacher's work and the student's learning.
\end{abstract}

Keywords: GeoGebra. Teaching. Learning. Students. Trigonometry 


\section{Introdução}

O trabalho com alunos do ensino médio nos mostra que muitos desses alunos apresentam dificuldades na compreensão de conceitos referentes à trigonometria. Compreender as características de funções como o seno e o cosseno ainda é um desafio para muitos alunos. $\mathrm{O}$ trabalho na licenciatura em Matemática confirma essa tendência, além de mostrar que muitos alunos concluem o Ensino Médio sem ao menos estudarem trigonometria.

Apesar da importância da trigonometria para o estudo das funções, da geometria e para a compreensão de conceitos da física clássica ${ }^{1}$, Oliveira (2006) ressalta que, nos seus 18 anos de trabalho com alunos do ensino fundamental e do Ensino Médio, comumente se deparava com alunos apresentando dificuldades nas aulas de trigonometria, ou mesmo na resolução de problemas da física envolvendo algum conceito trigonométrico.

Lopes (2011), a partir da prática em sala de aula da rede pública de ensino do Rio Grande do Norte $(\mathrm{RN})$ e das vivências em estudos, planejamentos, cursos de formação de professores, entre outros, mostra-nos que

Nessas vivências, em contato direto com colegas da área, evidenciamos que parte dos professores de Matemática do Ensino Médio das escolas públicas estaduais substituía conteúdos como trigonometria, logaritmos e números complexos, por considerá-los de difícil entendimento para os alunos, por uma revisão de temas já abordados anteriormente. Desse modo, o conteúdo de trigonometria fica relegado a um segundo plano. (LOPES, 2011, p. 1-2)

As dificuldades apresentadas pelos alunos na compreensão dos conceitos abordados no ensino de trigonometria evidenciam a necessidade de novas abordagens no ensino desses saberes.

Nesse sentido, diversos autores argumentam que a inserção do computador no ambiente escolar oferece possibilidades de melhoria do trabalho dos professores e da aprendizagem dos alunos, já que

${ }^{1}$ Como no caso do estudo de vetores e na decomposição de forças aplicadas a um corpo, onde são necessárias noções de seno e cosseno. as práticas educativas supõem processos comunicativos intencionais e os vínculos entre estes dois elementos (educação e comunicação) se estreitaram sensivelmente, na contemporaneidade.

As novas tecnologias podem reforçar a contribuição dos trabalhos pedagógicos e didáticos contemporâneos, pois permitem que sejam criadas situações de aprendizagem ricas, complexas, diversificadas, por meio de uma divisão de trabalho que não faz mais com que todo o investimento repouse sobre o professor, uma vez que tanto a informação quanto a dimensão interativa são assumidas pelos produtores dos instrumentos. (PERRENOUD, 2000, p. 139)

Lopes (2011) considera que as Tecnologias da Informação e da Comunicação (TIC) exercem um papel cada vez mais importante na educação, notadamente, na Educação Matemática. De acordo com o autor, pesquisas sobre o uso das TIC em sala de aula ressaltam a sua relevância no ensino de Matemática, assinalando que é de fundamental importância a sua presença na formação inicial dos professores.

Essa formação seria útil já no momento em que o professor escolhe os recursos tecnológicos que irá utilizar, pois, diante de tantas possibilidades de diversificação das atividades oferecidas pelas TIC, particularmente no uso de softwares no ensino de Matemática, cabe ao professor escolher adequadamente o software que irá utilizar, levando em consideração aspectos como: os objetivos traçados, o conteúdo abordado, as possibilidades de uso de recursos multimídia (som, imagens, vídeos etc.), a facilidade de instalação e utilização, os custos de aquisição etc.

Dentre as inúmeras possibilidades de uso da informática na educação e, mais especificamente, no ensino de Matemática, a utilização de softwares desenvolvidos com foco nos conhecimentos abordados por essa disciplina se apresenta como um meio importante para que os alunos brasileiros superem as imensas dificuldades na aprendizagem evidenciadas nas avaliações oficiais.

Os softwares de simulação envolvem a criação de modelos dinâmicos e simplificados 
do mundo real permitindo a exploração de progressos reais ou fictícios e os conduzindo a uma situação real de aprendizagem. A grande vantagem das simulações é a possibilidade de mudar e acrescentar dados e variáveis, manipulando assim os elementos que irão intervir na experiência. A simulação motiva respostas, a análise dos resultados e refina conceitos. (MERLO, ASSIS, 2010, p. 10)

Entre os diversos softwares desenvolvidos para auxiliar o ensino de Matemática (Cabri-Géomètre, Winplot, Wingeom etc.), deseja-se destacar o GeoGebra, tanto pelo fato de se tratar de um software cuja distribuição pública é livre, quanto pelas inúmeras possibilidades de utilização que esse software proporciona, nos variados temas tratados por essa área do conhecimento.

Criado por Markus Hohenwarter, o GeoGebra é um software livre de Matemática dinâmica desenvolvido para o ensino e aprendizagem da Matemática desde o ensino básico até o ensino universitário. Este software reúne recursos de geometria, álgebra, tabelas, gráficos, probabilidade, estatística e cálculos simbólicos em um único ambiente, com a vantagem didática de representar, ao mesmo tempo e em um único ambiente visual, as características geométricas e algébricas de um mesmo objeto.

Diante do imenso potencial pedagógico e da facilidade de aquisição do GeoGebra, é impossível não se cogitar as possibilidades de ampliação da aprendizagem oferecidas por esse software em conteúdos de Matemática onde os alunos apresentam sensíveis dificuldades de aprendizado, tais como a trigonometria.

Nesse sentido, apresenta-se os seguintes questionamentos:

a) O trabalho com o GeoGebra facilita a aprendizagem de conceitos da trigonometria?

b) Como os alunos avaliam a importância da utilização de softwares como o GeoGebra no ensino de Matemática?

Sem a pretensão de esgotar essa discussão, foi desenvolvida uma pesquisa sobre o uso do GeoGebra no ensino de trigonometria junto aos alunos do segundo ano do ensino médio integrado ao ensino técnico do campus de Mossoró do Instituto Federal de Educação, Ciência e Tecnologia do Rio Grande do Norte (IFRN), onde buscou-se atingir os seguintes objetivos:

a) Analisar se o trabalho com o GeoGebra facilita a aprendizagem de conceitos da trigonometria, tais como: crescimento e decrescimento das funções seno e cosseno no ciclo; determinação dos quadrantes onde os valores das imagens dessas funções são positivos ou negativos; comportamento dos seus gráficos e a influência dos parâmetros $a, b$ e $c$ no comportamento do período e da imagem das funções $f(x)=a \operatorname{sen}(b x)+c \quad \mathrm{e}$ $g(x)=a \cdot \cos (b x)+c$;

b) Analisar como os alunos avaliam a importância da utilização de softwares como o GeoGebra no ensino de Matemática.

Para que fosse possível compreender adequadamente a realidade analisada, foi desenvolvida uma pesquisa que combina aspectos da pesquisa qualitativa com aspectos quantitativos, fazendo uso de estratégias de coleta de dados variadas, tais como:

a) Atividades avaliativas antes e após a utilização do GeoGebra no ensino de trigonometria;

Através das atividades avaliativas, buscouse analisar se o GeoGebra facilitava a apreensão de características relativas ao comportamento das funções seno e cosseno.

b) Aplicação de entrevistas semiestruturadas com os alunos.

Através das entrevistas procurou-se conhecer as percepções dos alunos acerca do uso do GeoGebra no ensino de trigonometria e da importância da utilização desse software no ensino de Matemática.

Desse modo, foi adotado um modelo de pesquisa preocupado com a relação entre dados qualitativos, sistematização dos dados, quantificação e análise, em uma perspectiva que contemple as minúcias e sutilezas dos dados, sem negligenciar sua possibilidade de generalização.

Vejamos agora uma breve descrição das atividades desenvolvidas com o objetivo de analisar a importância do uso do GeoGebra para o trabalho com as funções seno e cosseno.

\section{A atividade com o GeoGebra}

Inicialmente, foram ministradas aulas expositivas sobre as funções seno e cosseno fazendo uso do livro didático e do quadro branco.

Depois de desenvolvidas essas atividades, foi realizada uma atividade avaliativa 
composta por 17 itens e que deveria ser respondida pelos alunos das três turmas que participaram da pesquisa.

Nessa avaliação, procurou-se analisar os níveis de compreensão de alguns conceitos explorados no ensino das funções seno e cosseno, tais como: o crescimento e decrescimento dessas funções; o comportamento dos seus gráficos; os quadrantes onde os valores da imagem dessas funções são positivos ou negativos; a determinação do período e da imagem dessas funções e a influência dos parâmetros a, b e c no comportamento do gráfico, da imagem e do período das funções $f(x)=$ a. $\operatorname{sen}(b x)+c$ e $g(x)=a \cdot \cos (b x)+c$.

De posse dos resultados dessa avaliação, foi estabelecido o limite de 12 acertos (aproximadamente 70\%) como sendo o mínimo necessário para que o aluno não precisasse fazer uma nova avaliação, de modo que todos os alunos que obtiveram menos de 12 acertos foram convidados a participar da segunda etapa da atividade, no laboratório de informática, já com o uso do GeoGebra.

Assim, após a apresentação dos resultados das avaliações e do agendamento dos dias e horários em que seriam realizadas as atividades com o GeoGebra, deu-se início à segunda etapa da atividade, já no laboratório de informática, onde os alunos puderam, em um primeiro momento, fazer uso do GeoGebra para construir o ciclo trigonométrico, identificando arcos, ângulos e os vetores que representariam os valores do seno e do cosseno.

Ao discutir as possibilidades oferecidas pelo uso da informática no ensino de Matemática, Loesch (2001) ressalta que a interatividade ${ }^{2}$ oportunizada por essa tecnologia desempenha um papel essencial no processo de ensinoaprendizagem, fazendo com que o aluno deixe de ser um mero expectador, aproveitando o potencial das possibilidades enriquecedoras oferecidas pelo uso dos múltiplos sentidos.

Por concordar com o autor supracitado e considerar que a possibilidade de interatividade direta com o software pode contribuir

2 Belloni (2003) define interatividade como uma característica técnica que significa a possibilidade de um usuário interagir com a máquina, diferente de interação, que é a ação recíproca entre dois ou mais atores onde ocorre intersubjetividade, isto é, encontro de dois ou mais sujeitos, podendo ser direta ou indireta (mediada por algum veículo de comunicação). positivamente para a aprendizagem, optou-se pela realização de uma atividade em que os alunos iriam interagir diretamente com o software, fazendo uso do GeoGebra, ao mesmo tempo em que as animações eram construídas e apresentadas, com o auxílio de um projetor multimídia.

Desse modo, todos os comandos utilizados na construção gradual da animação foram apresentados em uma projeção para que os alunos pudessem efetuar os mesmos comandos e visualizar as modificações na animação na tela do seu computador. Para facilitar a construção das animações, foi elaborado um tutorial com todos os passos a serem executados pelos alunos durante a atividade a ser desenvolvida no laboratório de informática.

No tutorial, todas as etapas necessárias à construção do ciclo trigonométrico foram apresentadas, com todos os elementos necessários à compreensão do comportamento das funções seno e cosseno (arcos, ângulos, projeções da extremidade do arco sobre os eixos etc.).

Depois de construída a animação, buscouse aproveitar a possibilidade de visualização dos objetos em movimento para promover uma discussão sobre o comportamento dessas funções no ciclo trigonométrico.

A imagem abaixo representa a apresentação onde se destaca o comportamento da função seno no ciclo trigonométrico.

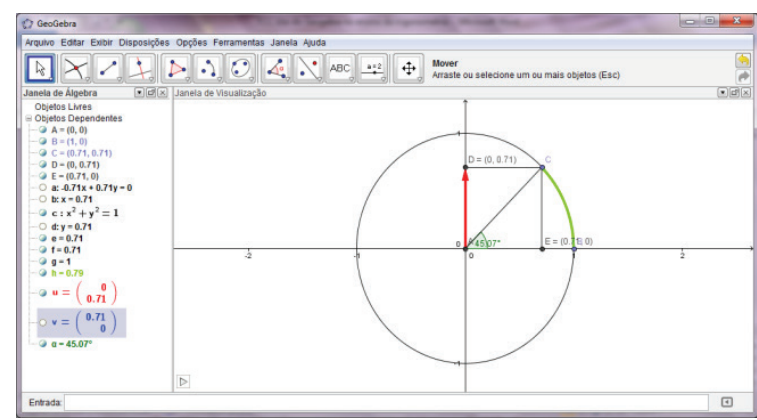

Figura 01 - A função seno no ciclo trigonométrico

Depois de apresentadas as modificações no comportamento da função seno, utilizou-se o recurso "exibir/esconder objeto" para ocultar o vetor referente ao valor do seno e exibir o vetor referente ao valor do cosseno.

A imagem abaixo representa a apresentação onde se destaca o comportamento da função cosseno no ciclo trigonométrico. 


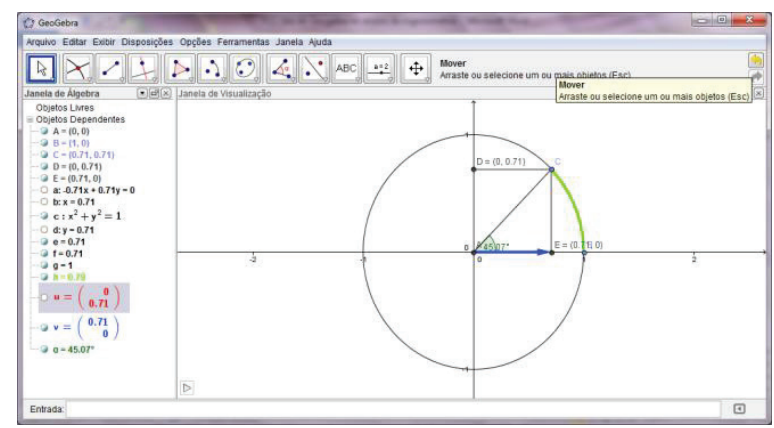

Figura 02 - A função cosseno no ciclo trigonométrico

Um aspecto importante a ser destacado acerca do uso do GeoGebra diz respeito à possibilidade de visualizar, simultaneamente ou separadamente, as modificações no comportamento das funções seno e cosseno.

A imagem abaixo representa a apresentação onde se destaca, ao mesmo tempo, o comportamento das funções seno e cosseno no ciclo trigonométrico.

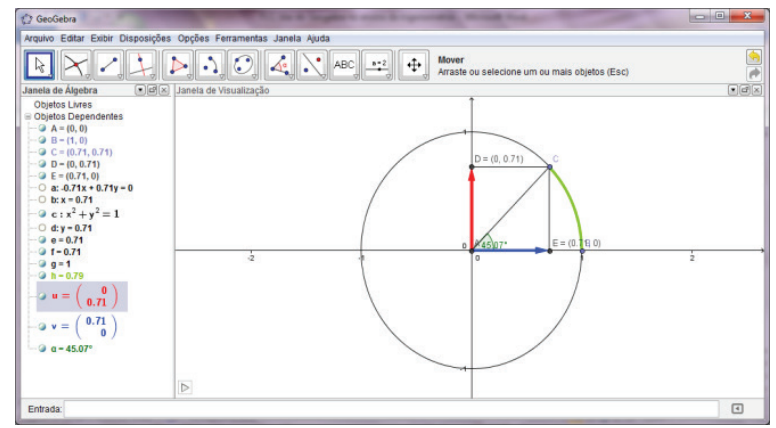

Figura 03 - As funções seno e cosseno no ciclo trigonométrico

Após a apresentação das características das funções seno e cosseno no ciclo trigonométrico, foram apresentadas as etapas necessárias à construção de alguns gráficos das funções $f(x)=a \cdot \operatorname{sen}(b x)+c$ e $g(x)=a \cdot \cos (b x)+c$, onde procurou-se realizar modificações nos parâmetros $a, b$ e $c$, para que os alunos pudessem compreender as implicações dessas modificações no comportamento dos gráficos, na amplitude, no período e na imagem dessas funções.

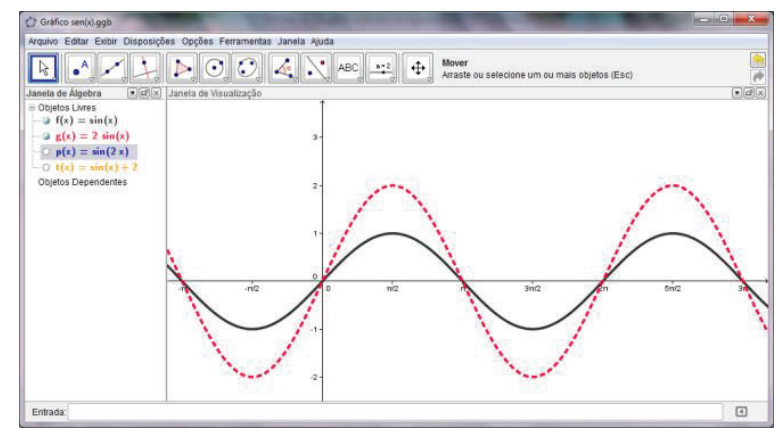

Figura 04 - Modificações no gráfico da função $f(x)=a \cdot \operatorname{sen}(b x)+c \quad$ ocasionadas por mudanças no parâmetro $a$ (dilatação vertical)

A função $f(x)=\operatorname{sen}(x)$ gera o gráfico da função $f(x)=a$. sen $(b x)+c$, onde $a$ vale $1, b$ vale 1 e $c$ vale 0 , determinando uma amplitude igual a 1 , um período igual a $2 \pi$ e uma imagem variando no intervalo [-1, 1]. Já a função $g(x)=2 \cdot \operatorname{sen}(x)$ origina o gráfico da função $f(x)=a$. sen $(b x)+c$, onde $a$ vale $2, b$ vale 1 e $c$ vale 0 , com amplitude igual a 2 , período igual a $2 \pi$ e imagem igual ao intervalo de $[-2,2]$.

Neste caso, a análise dos dois gráficos nos permite mostrar aos alunos que as modificações no parâmetro $a$ geram mudanças na amplitude e na imagem da função, enquanto que o período da função permanece o mesmo.

Através do uso do GeoGebra também foi possível mostrar aos alunos que as modificações que ocorreram na imagem e na amplitude da função seno, provocadas pelas modificações do parâmetro $a$, também irão ocorrer com a imagem e a amplitude da função cosseno, à medida que sejam promovidas modificações nos valores do parâmetro $a$, conforme se pode visualizar na figura abaixo.

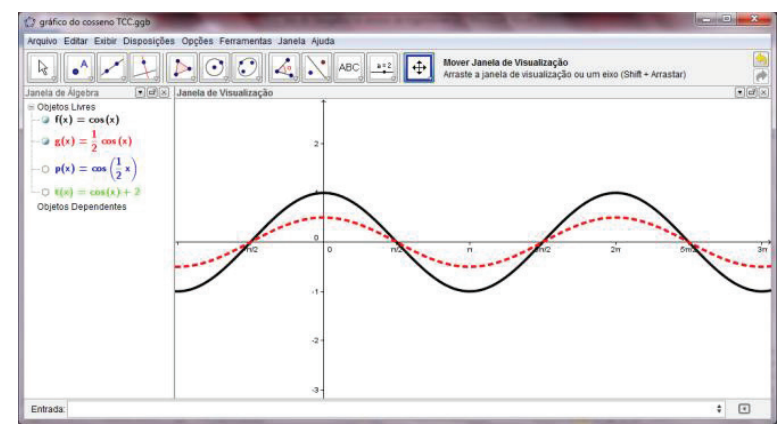

Figura 05 - Modificações no gráfico da função $f(x)=a \cdot \cos (b x)+c \quad$ ocasionadas por mudanças no parâmetro $a$ (compressão vertical) 
De modo análogo ao que ocorreu com a função seno, a análise dos gráficos das funções $f(x)=$ $\cos (x)$ e $g(x)=\frac{1}{2} \cdot \cos (x)$ permitiu mostrar aos alunos que as modificações no parâmetro $a$ geraram mudanças na amplitude e na imagem da função cosseno, enquanto que o período da função permaneceu o mesmo.

Outro exemplo importante é apresentado na imagem abaixo, onde foram apresentados os gráficos das funções $f(x)=\operatorname{sen}(x)$ e $\quad h(x)=$ $-\operatorname{sen}(x)$.

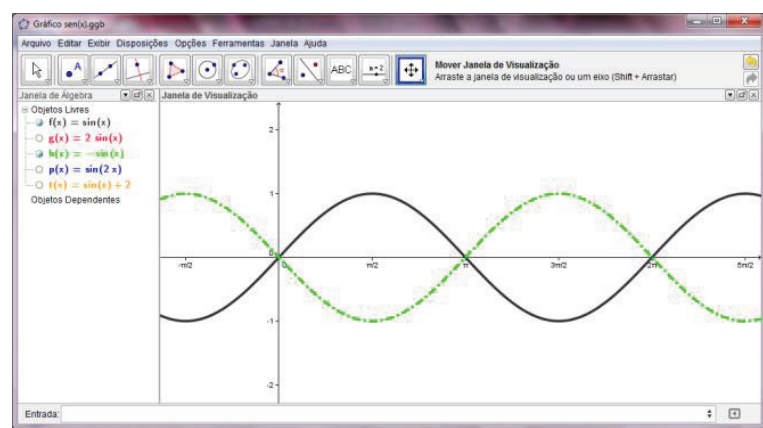

Figura 06 - Modificações no gráfico da função $f(x)=a \cdot \operatorname{sen}(b x)+c$ ocasionadas por mudanças no parâmetro $a$ (simetria)

Na figura acima, os gráficos das funções $f(x)=$ $\operatorname{sen}(x)$ e $h(x)=-\operatorname{sen}(x)$ evidenciam um exemplo de simetria, provocado pela mudança no sinal de $a$, onde, na função $f(x)$, o parâmetro $a$ vale 1 , enquanto que, na função $h(x)$, $a$ vale -1 .

Da mesma forma que o GeoGebra foi utilizado para demonstrar as modificações nos gráficos das funções $y=a \cdot \operatorname{sen}(b x)+c$ e $y=a \cdot \cos (b x)+c$, provocadas pela variação do parâmetro $a$, esse software foi utilizado para mostrar as modificações nos gráficos dessas funções ocasionadas pela variação nos parâmetros $b$ e $c$.

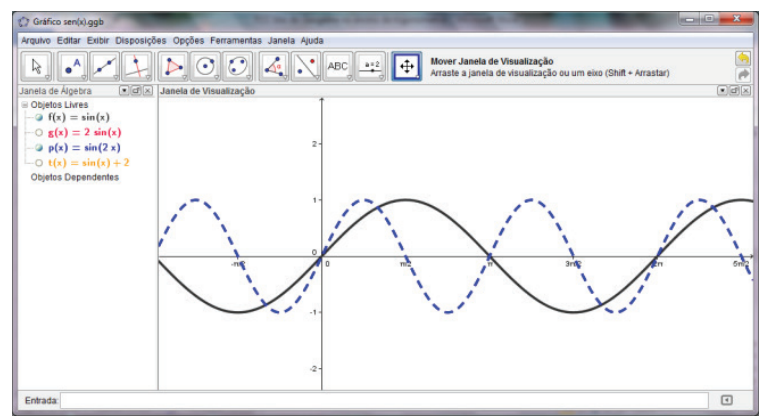

Figura 07 - Modificações no gráfico da função $f(x)$ $=a \operatorname{sen}(b x)+c$ ocasionadas por mudanças no parâmetro $b$ (compressão horizontal)
Neste caso, a análise dos dois gráficos permite mostrar aos alunos que as modificações no parâmetro $b$ geram mudanças no período da função, enquanto que a amplitude e a imagem da função permanecem as mesmas.

Mostrou-se ainda que as modificações que ocorreram no gráfico de $y=a \cdot \operatorname{sen}(b x)+c$, à medida que os valores de $b$ foram modificados, também ocorrem com a função $y=$ $a \cdot \cos (b x)+c$.

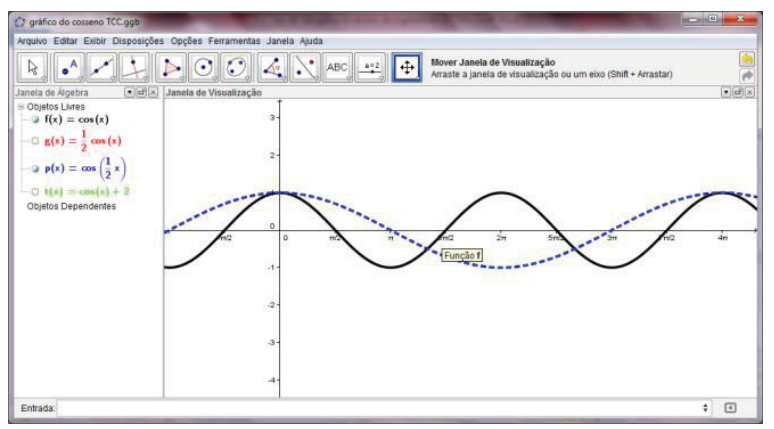

Figura 08 - Modificações no gráfico da função $f(x)=a \cdot \cos (b x)+c$ ocasionadas por mudanças no parâmetro $b$ (dilatação horizontal)

Da mesma forma que ocorreu com as funções $y=\operatorname{sen}(x)$ e $y=\operatorname{sen}(2 x)$ a análise dos gráficos das funções $f(x)=\cos (x)$ e $p(x)=\cos \left(\frac{1}{2} x\right)$ permitiu mostrar aos alunos que as modificações no parâmetro $b$ geraram mudanças no período da função cosseno, enquanto que a amplitude e a imagem da função permaneceram as mesmas.

Em seguida, usou-se o GeoGebra para ilustrar algumas das possíveis modificações que ocorrem no gráfico da função $f(x)=$ a. $\operatorname{sen}(b x)+c$, à medida que os valores de $c$ eram modificados.

Na figura abaixo, a função $f(x)=\operatorname{sen}(x)$ gera o gráfico da função $f(x)=a$. sen $(b x)+c$, onde $a$ vale $1, b$ vale 1 e $c$ vale 0 , com um período igual a $2 \pi$ e uma imagem variando no intervalo $[-1,1]$. Já a função $t(x)=\operatorname{sen}(x)+2$ gera o gráfico da função $f(x)=a$.sen $(b x)+c$, onde $a$ vale $1, b$ vale 1 e $c$ vale 2 , com um período igual a $2 \pi$ e uma imagem variando no intervalo $[1,3]$. 


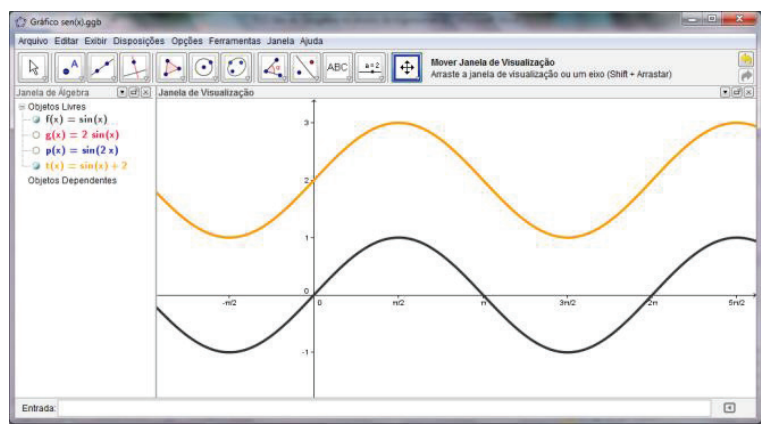

Figura 09 - Modificações no gráfico da função $f(x)=a$. sen $(b x)+c$ ocasionadas por mudanças no parâmetro $c$ (translação vertical)

De modo análogo ao que ocorreu com a função seno, os gráficos apresentados na figura abaixo mostram que as modificações no parâmetro $c$ geram mudanças na imagem da função $f(x)=$ a. $\cos (b x)+c$, enquanto que a amplitude (igual a 1) e o período da função (igual a $2 \pi$ ) permanecem os mesmos.

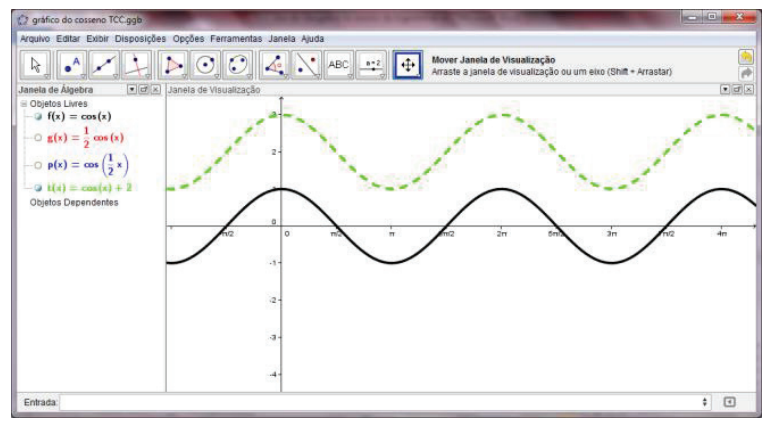

Figura 10 - Modificações no gráfico da função $f(x)=a \cdot \cos (b x)+c$ ocasionadas por mudanças no parâmetro $c$ (translação vertical)
Imediatamente após a realização da atividade com o GeoGebra, os alunos realizaram uma nova avaliação (sem o uso do GeoGebra), com a garantia de que a nota da primeira avaliação seria substituída pela nota desta nova avaliação, caso o aluno apresentasse alguma evolução no seu desempenho.

Vejamos agora uma análise do desempenho dos alunos a partir das respostas apresentadas nas duas avaliações, onde será possível perceber que, após o uso do GeoGebra, houve um número de acertos mais elevado quando comparado com o desempenho obtido na avaliação anterior, fato que é muito relevante, haja vista que apenas aqueles alunos com maiores dificuldades na compreensão dos temas abordados realizaram essa segunda avaliação.

\section{Análise do desempenho dos alunos antes e após o uso do GeoGebra no ensino de trigonometria}

A análise das avaliações realizadas antes e após a utilização do GeoGebra nos mostra que, em todas as turmas que participaram da atividade com esse software, ocorreu uma evolução significativa no desempenho dos alunos, conforme podemos observar na tabela 1.

Tabela 1: Desempenho dos alunos nas avaliações realizadas antes e após o uso do GeoGebra.

\begin{tabular}{l|c|c|c|l|l}
\hline Desempenho & Eletrotécnica & Informática & Mecânica & Total & $\%$ \\
\hline Fizeram a primeira avaliação & 37 & 39 & 34 & 110 & 100 \\
\hline Acima da nota de corte & 20 & 18 & 22 & 60 & 54,55 \\
\hline Abaixo da nota de corte & 17 & 21 & 12 & 50 & 45,45 \\
\hline Fizeram a segunda avaliação & 17 & 21 & 12 & 50 & 100 \\
\hline Melhoraram a nota & 11 & 18 & 12 & 41 & 82 \\
\hline Mantiveram a nota & 04 & 01 & 00 & 05 & 10 \\
\hline Pioraram a nota & 02 & 02 & 00 & 04 & 08 \\
\hline
\end{tabular}

Considerando-se que, antes da primeira avaliação, foram realizadas apenas atividades como: a exposição oral do conteúdo com o auxílio do quadro e do livro didático, a apresentação de exemplos e a resolução de exercícios propostos pelo livro, a constatação de que 60 alunos $(54,55 \%)$ conseguiram atingir ou superar os 12 acertos (aproximadamente 70\%) evidencia o bom nível de compreensão do comportamento das funções seno e cosseno por um número significativo de alunos, mesmo sem o uso de recursos considerados inovadores.

Apesar disso, a constatação de que 50 alunos $(45,45 \%)$ obtiveram um rendimento abaixo da nota de corte evidenciou as dificuldades 
apresentadas por vários desses alunos na compreensão do tema abordado.

Nesse contexto, tornam-se significativos os resultados apresentados pelos alunos na segunda avaliação, realizada após a utilização do GeoGebra, pois, entre os 50 alunos que participaram das atividades no laboratório e que fizeram essa avaliação, apenas 4 alunos (8,0\%) apresentaram uma redução nas suas notas com relação à avaliação anterior. Cinco alunos $(10,0 \%)$ mantiveram o mesmo desempenho e 41 alunos $(82,0 \%)$ melhoraram suas notas, o que evidencia a importância do uso do GeoGebra como um elemento que pode contribuir para a compreensão dos temas abordados.
Outro elemento importante a ser considerado é o fato de que, após o uso do GeoGebra, os alunos que apresentaram um desempenho insatisfatório na primeira avaliação obtiveram índices de acertos muito próximos aos índices de acertos apresentados pelos alunos que atingiram a nota de corte na primeira avaliação e, no caso dos alunos do curso de Mecânica, o índice de acertos foi superior ao índice apresentado pelos alunos que haviam obtido um desempenho satisfatório na primeira avaliação, conforme podemos constatar a partir dos dados apresentados na tabela 2 .

Tabela 2: Desempenho dos alunos que obtiveram a nota mínima na primeira avaliação e dos alunos que fizeram a segunda avaliação

\begin{tabular}{l|c|l|c|c}
\hline Curso & Acertos na primeira avaliação & $(\%)$ & Acertos na segunda avaliação & $(\%)$ \\
\hline Eletrotécnica & $262(20$ alunos $)$ & 77,06 & $203(17$ alunos $)$ & 70,24 \\
\hline Informática & $237(18$ alunos $)$ & 77,45 & $263(21$ alunos $)$ & 73,67 \\
\hline Mecânica & $303(22$ alunos $)$ & 81,02 & $178(12$ alunos $)$ & 87,25 \\
\hline Total & $802(60$ alunos $)$ & & 644 (50 alunos) &
\end{tabular}

A análise dos resultados das avaliações mostra que os 60 alunos que conseguiram atingir a nota mínima na primeira avaliação, obtiveram uma média de 13,37 acertos, enquanto que os 50 alunos que apresentaram um desempenho abaixo da nota mínima determinada para essa avaliação, obtiveram uma média de 8,96 acertos. Porém, estes alunos conseguiram alcançar uma média de 12,88 acertos na avaliação realizada após o trabalho com o GeoGebra.

Vejamos agora, mais detalhadamente, o desempenho dos alunos de cada um dos cursos nas avaliações aplicadas antes e após a utilização do GeoGebra, levando-se em consideração alguns dos saberes abordados nas atividades envolvendo as funções seno e cosseno.

Tabela 3: Número de acertos, por curso, nas questões envolvendo os sinais das funções seno e cosseno no ciclo, antes e após o uso do GeoGebra

\begin{tabular}{c|c|c|r|r|r|r|r|r}
\hline \multirow{2}{*}{ Questões } & \multicolumn{2}{|c|}{ Eletrotécnica } & \multicolumn{2}{c|}{ Informática } & \multicolumn{2}{c|}{ Mecânica } & \multicolumn{2}{c}{ Total } \\
\cline { 2 - 9 } & Antes & Depois & Antes & Depois & Antes & Depois & Antes & Depois \\
\hline 01 & 16 & 17 & 13 & 18 & 11 & 12 & 40 & 47 \\
\hline 05 & 15 & 15 & 09 & 18 & 06 & 12 & 30 & 45 \\
\hline $10 \mathrm{c}$ & 14 & 15 & 14 & 17 & 11 & 11 & 39 & 43 \\
\hline $10 \mathrm{~d}$ & 11 & 13 & 09 & 18 & 08 & 12 & 28 & 43 \\
\hline Total & 56 & 60 & 45 & 71 & 36 & 47 & 137 & 178 \\
\hline
\end{tabular}

Os dados apresentados na tabela acima mostram que, com exceção dos alunos do curso de Informática, os demais alunos apresentaram um índice de acertos satisfatório na primeira avaliação, o que evidencia que a maioria os alunos dos cursos de Eletrotécnica e Mecânica já apresentava uma boa compreensão do conteúdo abordado nessas questões, mesmo sem a utilização do GeoGebra.

$\mathrm{Na}$ primeira avaliação, enquanto que os alunos de Informática acertaram apenas 53,57\% das questões, os alunos de Mecânica e Eletrotécnica obtiveram índices de acertos de $75,0 \%$ e $82,35 \%$, respectivamente.

Após a utilização do GeoGebra, fica evidente a evolução no número de acertos, deixando clara a contribuição desse software para uma compreensão mais adequada da variação dos sinais das funções seno e cosseno no ciclo.

Vejamos agora o desempenho dos alunos nas questões envolvendo o crescimento e o 
decrescimento das funções seno e cosseno, antes e após a atividade com o GeoGebra.

Tabela 4: Número de acertos, por curso, nas questões envolvendo o crescimento e o decrescimento das funções seno e cosseno no ciclo, antes e após o uso do GeoGebra

\begin{tabular}{c|r|r|r|r|r|r|r|r}
\hline \multirow{2}{*}{ Questões } & \multicolumn{2}{|c|}{ Eletrotécnica } & \multicolumn{2}{c|}{ Informática } & \multicolumn{2}{c|}{ Mecânica } & \multicolumn{2}{c}{ Total } \\
\cline { 2 - 9 } & Antes & Depois & Antes & Depois & Antes & Depois & Antes & Depois \\
\hline 02 & 07 & 12 & 09 & 17 & 05 & 11 & 24 & 40 \\
\hline 06 & 03 & 12 & 04 & 17 & 01 & 11 & 08 & 40 \\
\hline
\end{tabular}

A tabela acima mostra que, na primeira curso de Mecânica, que passaram de cinco avaliação, os alunos apresentavam um nível de acertos (41,67\%) na questão envolvendo a função compreensão ainda precário acerca do seno e um acerto $(8,33 \%)$ na questão envolvendo crescimento e decrescimento das funções a função cosseno, para 11 acertos $(91,67 \%)$ em estudadas, principalmente no que concerne à função cosseno.

Após a utilização do GeoGebra, ambas as questões foram respondidas corretamente por 40 alunos $(80,0 \%)$, com destaque para os alunos do cada uma das questões.

Vejamos agora o desempenho dos alunos nas questões envolvendo o comportamento da imagem das funções seno e cosseno, antes e após a atividade com o GeoGebra.

Tabela 5: Número de acertos, por curso, nas questões envolvendo modificações na imagem das funções seno e cosseno, antes e após o uso do GeoGebra.

\begin{tabular}{c|r|r|r|r|r|r|r|r}
\hline \multirow{2}{*}{ Questões } & \multicolumn{2}{|c|}{ Eletrotécnica } & \multicolumn{2}{c|}{ Informática } & \multicolumn{2}{c|}{ Mecânica } & \multicolumn{2}{c}{ Total } \\
\cline { 2 - 9 } & Antes & Depois & Antes & Depois & Antes & Depois & Antes & Depois \\
\hline 03 & 14 & 14 & 17 & 16 & 08 & 09 & 39 & 39 \\
\hline 07 & 13 & 13 & 12 & 17 & 05 & 09 & 30 & 39 \\
\hline $09 \mathrm{a}$ & 13 & 12 & 17 & 19 & 07 & 12 & 37 & 43 \\
\hline $09 \mathrm{~b}$ & 07 & 15 & 13 & 19 & 03 & 12 & 23 & 46 \\
\hline
\end{tabular}

$\mathrm{Na}$ questão três, que abordava as modificações que ocorreram na imagem da função $f(x)=a$. sen $(b x)+c$, provocadas pelas mudanças no parâmetro $a$, os índices de acertos nas avaliações realizadas antes e após o uso do GeoGebra foram muito próximos, não evidenciando nenhuma evolução significativa devido ao uso desse software.

Já na questão sete, que abordava as modificações que ocorreram na imagem da função $g(x)=a \cdot \cos (b x)+c$, provocadas pelas mudanças no parâmetro $a$, os índices de acertos nas avaliações realizadas antes e após o uso do GeoGebra apresentaram uma evolução significativa nos cursos de informática $(41,67 \%)$ e Mecânica (80,0\%), permanecendo constante no curso de Eletrotécnica.

A questão (09a) que abordava as modificações no comportamento do gráfico da função $f(x)=a \cdot \operatorname{sen}(b x)+c$, apresentou índices de acertos muito próximos, nas avaliações realizadas antes e após o uso do GeoGebra, com exceção do curso de Mecânica, onde o número de acertos passou de sete $(58,33 \%)$ para 12 $(100,0 \%)$.

Quando se observa o desempenho dos alunos na questão que abordava as modificações que ocorreram no comportamento do gráfico da função $g(x)=a \cdot \cos (b x)+c$, fica evidente que os alunos dos três cursos apresentaram uma evolução significativa no número de acertos na avaliação realizada após o uso do GeoGebra, especialmente no curso de Mecânica, onde os alunos passaram de apenas três acertos $(25,0 \%)$ na primeira avaliação, para 12 acertos $(100,0 \%)$ na segunda avaliação, evidenciando que o uso desse software ajuda a corrigir essa deficiência no aprendizado das características da função cosseno.

Quando se fala em deficiências no aprendizado das características das funções seno e cosseno, não se pode esquecer a dificuldade que os alunos apresentaram na compreensão do comportamento do período dessas funções.

Nas atividades em sala de aula, já era possível perceber a dificuldade que alguns alunos apresentavam para determinar o período das funções seno e cosseno. Após a realização da primeira avaliação, o péssimo desempenho apresentado pelos alunos nas questões que 
discutem o período dessas funções deixa evidente essa dificuldade.

Tabela 6: Número de acertos, por curso, nas questões envolvendo modificações no período das

funções seno e cosseno, antes e após o uso do GeoGebra

\begin{tabular}{c|c|c|r|r|r|r|r|r}
\hline \multirow{2}{*}{ Questões } & \multicolumn{2}{|c|}{ Eletrotécnica } & \multicolumn{2}{c|}{ Informática } & \multicolumn{2}{c|}{ Mecânica } & \multicolumn{2}{c}{ Total } \\
\cline { 2 - 9 } & Antes & Depois & Antes & Depois & Antes & Depois & Antes & Depois \\
\hline 04 & 01 & 09 & 02 & 16 & 02 & 09 & 05 & 34 \\
\hline 08 & 03 & 07 & 03 & 10 & 03 & 10 & 09 & 27 \\
\hline $09 \mathrm{c}$ & 12 & 15 & 11 & 17 & 04 & 11 & 28 & 43 \\
\hline $09 \mathrm{~d}$ & 06 & 12 & 07 & 14 & 04 & 09 & 18 & 35 \\
\hline
\end{tabular}

Nas questões de múltipla escolha (questões influência sobre o período das funções seno e 04 e 08) o número de acertos é bem menor que o número de acertos nas questões em que o aluno precisava apenas dizer se a afirmação era verdadeira ou falsa (9c e 9d), o que é perfeitamente compreensível, haja vista que, mesmo sem conhecer efetivamente a influência dos parâmetros $a, b$ e $c$ no comportamento do período das funções, a probabilidade de acertar questões com apenas duas opções de resposta (50,0\%) é bem maior do que a probabilidade de acertar questões com cinco opções de resposta (20,0\%).

Além disso, como as questões 9c e 9d objetivavam apenas saber se o aluno era capaz de identificar qual dos parâmetros exerce cosseno, enquanto que as questões quatro e oito levavam o aluno a refletir sobre como se davam essas modificações no período, é possível que alguns alunos soubessem qual dos parâmetros interfere no período das funções, sem saber exatamente de que maneira isso ocorre.

Após o uso do GeoGebra, fica evidente a evolução no número de acertos entre os alunos de todos os cursos, tanto nas questões de múltipla escolha, quanto nas questões em que o aluno era questionado se a afirmação era verdadeira ou falsa.

Vejamos agora o desempenho dos alunos nas questões que abordavam o comportamento dos gráficos das funções seno e cosseno.

Tabela 7: Número de acertos, por curso, nas questões envolvendo o comportamento dos gráficos das funções seno e cosseno, antes e após o uso do GeoGebra.

\begin{tabular}{c|c|c|r|r|r|r|r|r}
\hline \multirow{2}{*}{ Questões } & \multicolumn{2}{|c|}{ Eletrotécnica } & \multicolumn{2}{c|}{ Informática } & \multicolumn{2}{c|}{ Mecânica } & \multicolumn{2}{c}{ Total } \\
\cline { 2 - 9 } & Antes & Depois & Antes & Depois & Antes & Depois & Antes & Depois \\
\hline $09 \mathrm{e}$ & 13 & 09 & 16 & 14 & 03 & 11 & 32 & 34 \\
\hline $10 \mathrm{a}$ & 12 & 07 & 14 & 09 & 04 & 09 & 30 & 25 \\
\hline $10 \mathrm{~b}$ & 12 & 06 & 13 & 07 & 08 & 08 & 33 & 21 \\
\hline
\end{tabular}

Enquanto que a questão 9e tratava da $f(x)=a \cdot \operatorname{sen}(b x)+c$ e $g(x)=a \cdot \cos (b x)+c$ influência do parâmetro $c$ no comportamento das funções seno e cosseno, as questões 10a e 10b buscavam verificar se os alunos eram capazes de identificar onde os gráficos das funções seno e cosseno interceptavam o eixo $\mathrm{x}$.

Surpreendentemente, os alunos dos cursos de Eletrotécnica e Informática apresentaram um desempenho pior na avaliação realizada após o uso do GeoGebra, do que na avaliação realizada antes do trabalho com esse software, especialmente nas questões que tratavam dos valores onde os gráficos das funções seno e cosseno interceptavam o eixo x. Apenas os alunos do curso de Mecânica conseguiram apresentar um evolução significativa no número de acertos após a atividade com o GeoGebra.

A dificuldade apresentada nas questões que envolvem o comportamento dos gráficos de demonstra a importância do desenvolvimento de atividades complementares que estimulem o aluno a construir os gráficos dessas funções e a refletir sobre as modificações sofridas por esses gráficos, à medida que os valores dos parâmetros $a, b$ e $c$, são modificados.

Nunca é demais lembrar que o GeoGebra fornece o gráfico pronto, logo após a digitação da função na caixa de entrada, sem que o aluno faça uma reflexão mais apurada acerca de como aquele gráfico foi construído.

Se essa rapidez é importante no momento em que se deseja comparar gráficos de funções, ela deixa a desejar no que diz respeito à possibilidade de o aluno escolher os valores que irá atribuir à variável $x$ e constatar os consequentes valores encontrados para a 
variável $y$, formando os pares ordenados $(x, y)$ que irão compor o gráfico.

Nesse sentido, a utilização do livro didático pode exercer uma contribuição importante, por trazer vários exemplos onde são feitas construções detalhadas dos gráficos dessas funções, além de exercícios que estimulam o aluno a construir, passo a passo, esses gráficos.

Além da análise das contribuições do uso do GeoGebra para a aprendizagem de conceitos da trigonometria envolvendo as funções seno e cosseno, também buscou-se saber quais as percepções dos alunos acerca do uso de softwares como o GeoGebra no ensino de Matemática.

\section{Percepções dos alunos acerca do uso do GeoGebra no ensino de trigonometria}

Através da entrevista, os alunos foram questionados acerca da importância da utilização de softwares como o GeoGebra no ensino de trigonometria.

Tabela 8: Percepções dos alunos acerca da importância da utilização do GeoGebra no ensino de trigonometria.

\begin{tabular}{l|l|l|l|l}
\hline Grau de importância & Eletrotécnica & Informática & Mecânica & Total \\
\hline Muito importante & 08 & 10 & 08 & 26 \\
\hline Importante & 05 & 08 & 03 & 16 \\
\hline Razoavelmente importante & 0 & 1 & 0 & 01 \\
\hline Pouco importante & 1 & 0 & 0 & 01 \\
\hline Nenhuma importância & 0 & 0 & 0 & 0 \\
\hline
\end{tabular}

A recepção positiva por parte dos alunos à ideia de utilizar softwares como o GeoGebra no ensino de trigonometria fica evidente, não só pelo elevado número de alunos que considera esse uso importante $(36,36 \%)$ ou muito importante $(59,09 \%)$, como também nas afirmações feitas por esses alunos ao justificar suas opiniões.

Como já era previsível, diante de tudo o que foi apresentado até o momento, a quase totalidade dos alunos (88,64\%) justifica a importância da utilização do GeoGebra afirmando que o uso deste software facilita a aprendizagem dos conteúdos.

Mais do que simplesmente afirmar que o uso do GeoGebra facilita a aprendizagem, alguns desses alunos destacam aspectos importantes e que contribuem para essa facilitação, dentre os quais pode-se citar: a possibilidade de desenvolver uma atividade prática que ajuda a fixar a aprendizagem (15,91\%), ou ainda, a facilidade na visualização, já que a apresentação se torna mais minuciosa e clara $(22,73 \%)$.

Porque o GeoGebra abre o campo de visão do aluno na Matemática. Ele mostra, na prática, o que é ensinado na sala de aula, a qual é um ambiente que não possibilita uma visão muito aberta sobre vários assuntos. O GeoGebra também ajuda muito na compreensão dos alunos, pois ele mostra detalhadamente o comportamento da função, o qual não fica muito claro quando é ensinado em sala de aula, pois o instrumento de trabalho usado não deixa muito claro o que, na realidade, o professor quer passar para o aluno. (Aluno 01)

Outros aspectos importantes sobre a utilização do GeoGebra apontados pelos alunos destacam o fato de que o uso desse software melhora o trabalho do professor $(6,82 \%)$, torna a aula mais dinâmica $(6,82 \%)$, cativa e proporciona mais liberdade ao aluno (9,09\%), de modo que a aprendizagem se dá de forma mais rápida $\mathrm{e}$ prazerosa $(4,54 \%)$.

Não sei como está a situação nas outras escolas em relação a esse assunto, mas aqui é uma inovação, uma evolução daquelas aulas chatas, torna as aulas mais dinâmicas. Todos os alunos que utilizaram o programa disseram que aprenderam mais com essa aula do que o resto das aulas sobre trigonometria, por causa da liberdade que o software proporciona. (Aluno 02)

A afirmação acima aponta ainda para dois aspectos essenciais acerca do uso de softwares no ensino de Matemática: por um lado, uma evolução nas possibilidades de aprendizagem e, por outro lado, o questionamento dos métodos de ensino de Matemática comumente utilizados. 


\section{Conclusões}

Ao longo desta pesquisa procurou-se analisar se o trabalho com o GeoGebra facilita a aprendizagem de conceitos da trigonometria envolvendo as funções seno e cosseno, e ainda analisar as percepções dos alunos acerca da importância da utilização de softwares como o GeoGebra no ensino de Matemática.

Para que fosse possível compreender adequadamente a realidade analisada, foi realizada uma atividade prática com o uso do GeoGeobra no ensino de trigonometria, com foco nas características das funções seno e cosseno, bem como atividades avaliativas antes e após a utilização desse software.

Além disso, foram realizadas entrevistas semiestruturadas com os alunos que participaram da atividade com o objetivo de conhecer as percepções desses alunos acerca da utilização do GeoGebra no ensino de trigonometria e da importância da utilização desse software no ensino de Matemática.

Os resultados obtidos a partir das atividades desenvolvidas junto aos alunos do segundo ano do ensino médio integrado ao ensino técnico do campus de Mossoró do IFRN nos mostraram que o uso do GeoGebra trouxe uma contribuição significativa para a aprendizagem de diversos aspectos inerentes ao comportamento das funções seno e cosseno.

Além disso, os alunos apresentaram percepções bastante positivas acerca da importância de se utilizar softwares como o GeoGebra no ensino de Matemática, ressaltando aspectos como: a possibilidade de desenvolver atividades práticas que ajudam a fixar a aprendizagem; a maior dinamicidade da aula; a melhoria no trabalho do professor; o maior envolvimento dos alunos; a possibilidade de observar os objetos em movimento e a facilidade na visualização desses objetos.

A evolução na aprendizagem após o uso do GeoGebra foi tão significativa que os alunos que participaram da atividade com esse software obtiveram índices de acertos muito próximos, ou até superiores, aos índices de acertos apresentados pelos alunos que atingiram a nota de corte na primeira avaliação.

É importante ressaltar que, apesar da evolução apresentada pelos alunos após a atividade com o GeoGebra, as dificuldades apresentadas na compreensão de alguns aspectos do comportamento das funções seno e cosseno ${ }^{3}$ evidenciaram que a utilização desse software não elimina a necessidade de utilização de outros meios de abordagem do conhecimento (livro didático, paradidáticos, vídeos etc.).

Uma formação adequada dos professores é outro aspecto fundamental para que o uso dos recursos computacionais na educação ocorra de modo adequado, pois, sem uma formação consistente, que seja capaz de apresentar-lhes as tecnologias e as suas implicações na educação e na sociedade, muitos desses profissionais continuaram se esquivando das TIC ou ainda fazendo usos inadequados desses recursos.

É comum ouvir críticas ao trabalho dos professores onde se afirma que esses profissionais precisam romper com práticas de ensino tradicionais e incorporar as TIC às suas atividades. Porém, considera-se que de nada adianta criticar o trabalho dos professores, sem que se desenvolvam políticas de formação que modifiquem essa situação.

Essa formação para o uso das TIC deveria se dar desde a formação inicial, na licenciatura, estendendo-se para os espaços de formação continuada, que poderiam ocorrer no interior da própria escola em que o professor atua, bem como nos cursos de pós-graduação.

Analisando especificamente $\mathrm{o}$ trabalho desenvolvido pelo professor de Matemática, é possível perceber que essa formação o ajudaria a promover uma proposta de ensino voltada para um uso consciente das TIC, distanciado dos modismos tão comuns na educação, estimulando a curiosidade e a criatividade do aluno, motivando-o a participar mais ativamente do processo ensino-aprendizagem, favorecendo a compreensão dos temas abordados e despertando sua paixão pela Matemática.

${ }^{3}$ Ver os dados apresentados na Tabela 07, com o desempenho dos alunos nas questões que tratam da influência do parâmetro $c$ no comportamento das funções seno e cosseno e as questões que buscavam verificar se os alunos eram capazes de identificar onde os gráficos das funções seno e cosseno interceptavam o eixo $\mathrm{x}$. 


\section{Agradecimentos}

Agradecemos a Coordenação de Aperfeiçoamento de Pessoal de Nível Superior (CAPES) pelo financiamento desta pesquisa.

\section{Referências}

BELLONI, Maria Luiza. Educação a distância. 3. ed. Campinas-SP: Editora Autores Associados, 2003.

LOESCH, Claudio. A informática na Matemática. In: GAERTNER, Rosinete (Org.). Tópicos de Matemática para o ensino médio. BlumenauSC: Edifurb, 2001, p. 23-38. Coleção Arithmos.

LOPES, Maria. Maroni. Contribuições do software GeoGebra no ensino e aprendizagem de trigonometria. XIII Conferência Interamericana de Educação Matemática. Recife, 2011.

MERLO, Clinton André, ASSIS, Raquel Trindade de. $O$ uso da informática no ensino da Matemática. Revista UNIJALES. Ed. 4, n. 4, ano $\mathrm{V}, 2010$.

OLIVEIRA, Francisco Canindé de. Dificuldades no processo ensino aprendizagem de trigonometria por meio de atividades. $74 \mathrm{f}$. Dissertação (Mestrado em Ensino de Ciências Naturais e Matemática) - Universidade Federal do Rio Grande do Norte, Natal, 2006.

PERRENOUD, Philippe. 10 novas competências para ensinar. Trad. Patrícia Chittoni Ramos. Porto Alegre: Artes Médicas, 2000. Capítulo 8, Utilizar novas tecnologias. 